\title{
3D Position Tracking in Challenging Terrain
}

\author{
Pierre Lamon and Roland Siegwart \\ Ecole Polytechnique Fédérale de Lausanne \{firstname.lastname\}@epfl.ch
}

\begin{abstract}
Summary. The intent of this paper is to show how the accuracy of 3D position tracking can be improved by considering rover locomotion in rough terrain as a holistic problem. An appropriate locomotion concept endowed with a controller minimizing slip improves the climbing performance, the accuracy of odometry and the signal/noise ratio of the onboard sensors. Sensor fusion involving an inertial measurement unit, 3D-Odometry, and visual motion estimation is presented. The experimental results show clearly how each sensor contributes to increase the accuracy of the $3 \mathrm{D}$ pose estimation in rough terrain.
\end{abstract}

\section{Introduction}

In order to acquire knowledge about the environment, a mobile robot uses different types of sensors, which are error prone and whose measurements are uncertain. In office-like environments, the interpretation of this data is facilitated thanks to the numerous assumptions that can be formulated e.g. the soil is flat, the walls are perpendicular to the ground, etc. In natural scenes, the problem is much more tedious because of limited a priori knowledge about the environment and the difficulty of perception. In rough terrain, the change in lighting conditions can strongly affect the quality of the acquired images and the vibrations due to uneven soils lead to noisy sensor signals. When the robot is overcoming an obstacle, the field of view can change significantly between two data acquisitions, increasing the difficulty of tracking features in the scene.

To get a robust estimate of the robots position, the measurements acquired by several complementary sensors have to be fused accounting for their relative variance. In the literature, the localization task generally involves two types of sensors and is divided into two phases a) the first step consists in the integration of a high frequency dead reckoning sensor to predict vehicle location b) the second phase, which is usually activated at a much slower rate, uses an absolute sensing mechanism for extracting relevant features in the environment and updating the predicted position. In [1], an inertial measurement 
unit is used for the prediction and an omnicam is used as the exteroceptive sensor. The pair of sensors composed of an inertial measurement unit and a GPS is used in [2]. Even if sensor fusion can be applied to combine the measurements acquired by any number of sensors, most of the applications found in the literature generally use only two types of sensors and only the 2D case is considered (even for terrestrial rovers).

In challenging environments, the six degrees of freedom of the rover have to be estimated (3D case) and the selection of sensors must be done carefully because of the aformentioned difficulties of perception in rough terrain. However, the accuracy of the position estimates does not only depend on the quality and quantity of sensors mounted onboard but also on the specific locomotion characteristics of the rover and the way it is driven. Indeed, the sensor signals might not be usable if an unadapted chassis and controller are used in challenging terrain. For example, the ratio signal/noise is poor for an inertial measurement unit mounted on a four-wheel drive rover with stiff suspensions. Furthermore, odometry provides bad estimates if the controller does not include wheel-slip minimization or if the kinematics of the rover is not accounted for.

The intent of this paper is to show how the accuracy of 3D position tracking can be improved by considering rover locomotion in rough terrain as a holistic problem. Section 2 describes the robotic platform developed for conducting this research. In Sect. 3, a method for computing 3D motion increments based on the wheel encoders and state sensors is presented. Because it accounts for the kinematics of the rover, this method provides better results than the standard method. Section 4 proposes a new approach for slip-minimization in rough terrain. Using this controller, both the climbing performance of the rover and the accuracy of the odometry are improved. Section 5 presents the results of the sensor fusion using 3D-Odometry, an Inertial Measurement Unit (IMU) and Visual Motion Estimation based on stereovision (VME). The experiments show clearly how each sensor contributes to increase the accuracy and robustness of the 3D pose estimation. Finally, Sect. 6 concludes this paper.

\section{Research platform}

The Autonomous System Lab (at EPFL) developed a six-wheeled off-road rover called Shrimp, which shows excellent climbing capabilities thanks its passive mechanical structure [3]. The most recent prototype, called SOLERO, has been equipped with sensors and more computational power (see Fig. 1). The parallel architecture of the bogies and the spring suspended fork provide a high ground clearance while keeping all six motorized wheels in ground-contact at any time. This ensures excellent climbing capabilities over obstacles up to two times the wheel diameter and an excellent adaptation to all kinds of terrains. The ability to move smoothly across rough terrain has many advantages when dealing with onboard sensors: for example, it allows limited wheel slip 
and reduces vibration. The quality of the odometric information and the ratio signal/noise for the inertial sensors are significantly improved in comparison with rigid structures such as four-wheel drive rovers. Thus, both odometry and INS integration techniques can be accounted for position estimation.
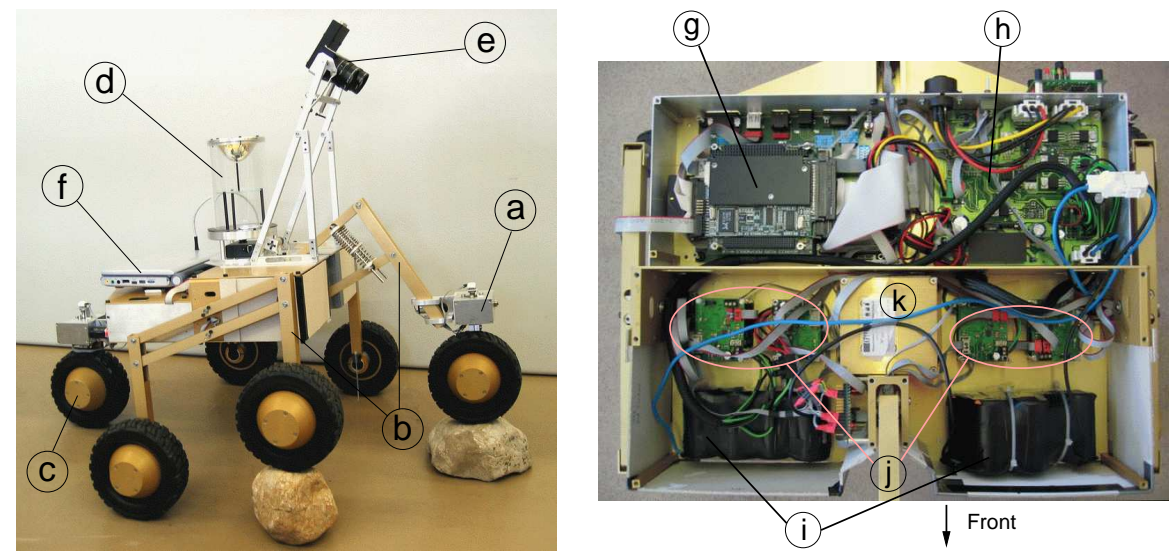

Fig. 1: Sensors, actuators and electronics of SOLERO. a) steering servo mechanism b) passively articulated bogie and spring suspended front fork (equipped with angular sensors) c) 6 motorized wheels (DC motors) d) omnidirectional vision system e) stereo-vision module, orientable around the tilt axis f) laptop (used for image processing) g) low power pc104 (used for sensor fusion) h) energy management board i) batteries (NiMh $7000 \mathrm{mAh}$ ) j) $\mathrm{I}^{2} \mathrm{C}$ slave modules (motor controllers, angular sensor module, servo controllers etc.) k) IMU (provides also roll and pitch)

\section{3D-Odometry}

Odometry is widely used to track the position and the orientation $\left([x, y, \psi]^{T}\right)$ of a robot in a plane $\pi$. This vector is updated by integrating small motion increments between two subsequent robot poses. This $2 \mathrm{D}$ odometry method can be extended in order to account for slope changes in the environment and to estimate the $3 \mathrm{D}$ position in a global coordinate system i.e. $[x, y, z, \phi, \theta, \psi]^{T}$. This technique uses typically an inclinometer for estimating the roll $(\phi)$ and pitch $(\theta)$ angles relative to the gravity field [4]. Thus, the orientation of the plane $\pi$, on which the robot is currently moving, can be estimated. The, $\mathrm{z}$ coordinate is computed by projecting the robot displacements in $\pi$ into the global coordinate system. This method, which will be referred later as the standard method, works well under the assumption that the ground is relatively smooth and does not have too many slope discontinuities. Indeed, the system accumulates errors during transitions because of the planar assumption. In 
rough terrain, this assumption is not verified and the transitions problem must be addressed properly. This section briefly describes a new method, called 3D-Odometry, which takes the kinematics of the robot into account and treats the slope discontinuity problem. The main reference frames and some of the variables used for 3D-Odometry are introduced in Fig. 2

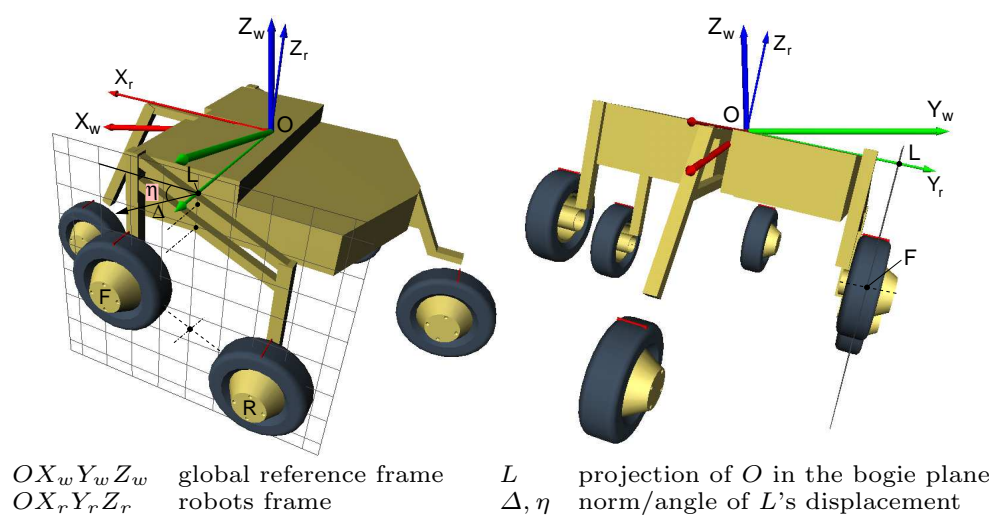

Fig. 2: Reference frames definition

The norm $\Delta$ and the direction of motion $\eta$ of each bogie can be computed by considering the kinematics of the bogie, the incremental displacement of the Rear/Front bogie wheels (wheel encoders) and the angular change of the bogie (angular sensor) between two data acquisition cycles. Then, the displacement of the robot's center $O$, i.e. $[x, y, z, \psi]^{T}$, can be computed using $\Delta$ and $\eta$ of the left and the right bogie, whereas the attitude $[\phi, \theta]^{T}$ is directly given by the inclinometer ${ }^{1}$.

\section{Experimental results}

The robot has been driven across obstacles of known shape and the trajectory computed online with both 3D-Odometry and the standard method. In all the experiments, the 3D-Odometry produced much better results than the standard method because the approach accounts for the kinematics of the rover. The difference between the two techniques becomes bigger as the difficulty of the obstacles increases (see Fig. 3). In Fig. 4, an experiment testing the full $3 \mathrm{D}$ capability of the method is depicted. The position error at the goal is only $\epsilon_{x}=1.4 \%, \epsilon_{y}=2 \%, \epsilon_{z}=2.8 \%, \epsilon_{\psi}=4 \%$ for a total path length of around $2 \mathrm{~m}$.

SOLERO has a non-hyperstatic mechanical structure that yields a smooth trajectory in rough terrain. As a consequence wheel slip is intrinsically minimized. When combined with 3D-Odometry, such a design allows to use odom-

\footnotetext{
${ }^{1}$ The reader can refer to the original paper [5] for more details about 3D-Odometry. In particular, the method also computes the wheel-ground contact angles.
} 
etry as a mean to track the rover's position in rough terrain. Moreover, the quality of odometry can still be significantly improved using a "smart" controller minimizing wheel slip. Its description is presented in the next section.

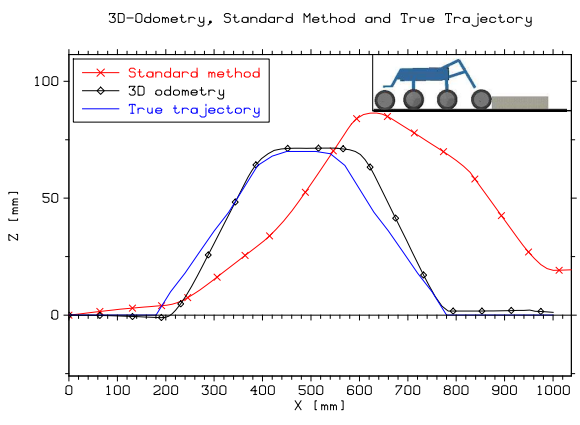

Fig. 3: Sharp edges experiment

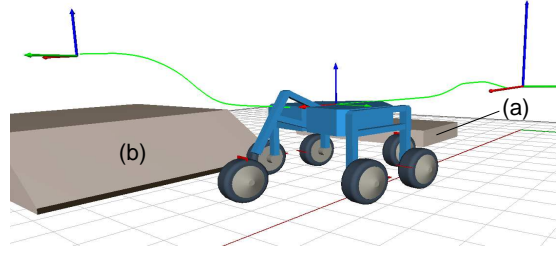

Only the right bogie wheels climbed obstacle (a). Then, the rover has been driven over obstacle (b) (with an incident angle of approximatively $20^{\circ}$ )

Fig. 4: Full 3D experiment

\section{Wheel slip minimization}

For wheeled rovers, the motion optimization is somewhat related to minimizing wheel slip. Minimizing slip not only limits odometric error but also increases the robot's climbing performance and efficiency. In order to fulfill this goal, several methods have been developed.

Methods derived from the Anti-lock Breaking System can be used for rough terrain rovers. Because they adapt the wheel speeds when slip already occurred, they are referred to as reactive approaches. A velocity synchronization algorithm, which minimizes the effect of the wheels fighting each other, has been implemented on the NASA FIDO rover [6]. The first step of the method consists in detecting which of the wheels are deviating significantly from the nominal velocity profile. Then a voting scheme is used to compute the required velocity set point change for each individual wheel. However, performance might be improved by considering the physical model of the rover and wheel-soil interaction models for a specific type of soil. Thus, the traction of each wheel is optimized considering the load distribution on the wheels and the soil properties. Such approaches are referred to as predictive approaches.

In [7], wheel-slip limitation is obtained by minimizing the ratio $T / N$ for each wheel, where $T$ is the traction force and $N$ the normal force. Reference [8] proposes a method minimizing slip ratios and thus avoid soil failure due to excessive traction. These physics-based controllers assume that the parameters of the wheel-ground interaction models are known. However, these parameters are difficult to estimate and are valid only for a specific type of soil and condition. Reference [9] proposes a method for estimating the soil parameters as the 
robot moves, but it is limited to a rigid wheel travelling through deformable terrain. In practice, the rover wheels are subject to roll on different kind of soils, whose parameters can change quickly. Thus, physics-based controllers are sensitive to soil parameters variation and difficult to implement on real rovers. In this section, a predictive approach considering the load distribution on the wheels and which does not require complex wheel-soil interaction models is presented. More details about the controller can be found in [10].

\section{Quasi-static model}

The speed of an autonomous rover is limited in rough terrain because the navigation algorithms are computationally expensive (limited processing power) and for safety reasons. In this range of speeds, typically smaller than $20 \mathrm{~cm} / \mathrm{s}$, the dynamic forces might be neglected and a quasi-static model is appropriate. To develop such a model, the mobility analysis of the rover's mechanical structure has to be done. It ensures to produce a consistent physical model with the appropriate degrees of freedom at each joints. Then the forces are introduced and the equilibrium equations are written for each part composing the rover's chassis. Because we have no interest in implicitly calculating the internal forces of the system, it is possible to reduce this set of independent equations. The variables of interest are the 3 ground contact forces on the front and the back wheel, the 2 ground contact forces on each wheel of the bogies and the 6 wheel torques. This makes 20 unknowns of interest and the system can be reduced to 15 equations. This leads to the following equation

$$
M_{15 x 20} \cdot \mathbf{U}_{20 x 1}=\mathbf{R}_{15 x 1}
$$

where $M$ is the model matrix depending on the geometric parameters and the state of the robot, $\mathbf{U}$ a vector containing the unknowns and $\mathbf{R}$ a constant vector. It is interesting to note that there are more unknowns than equations in 1 . That means that there is an infinite set of wheel-torques guaranteeing the static equilibrium. This characteristic is used to control the traction of each wheel and select, among all the possibilities, the set of torques minimizing slip. The optimal torques are selected by minimizing the function

$$
f=\max \left(\sum_{i} T_{i} / N_{i}\right) \quad i=1 . .6
$$

where $T_{i}$ and $N_{i}$ are the traction and the normal force applied to wheel $i$.

\section{Rover motion}

A static model balances the forces and moments on a system to remain at rest or maintain a constant speed. Such a system is an ideal case and does not include resistance to movement. Therefore, an additional torque compensating the rolling resistance torque must be added on the wheels in order to complete 
the model and guarantee motion at constant speed. This results in a quasistatic model. Unlike the other approaches, we don't use complex wheel-soils interaction models. Instead, we introduce a global speed control loop, in order to estimate the rolling resistance as the robot moves. The final controller, minimizing wheel slip and including rolling resistance, is depicted in Fig. 5.

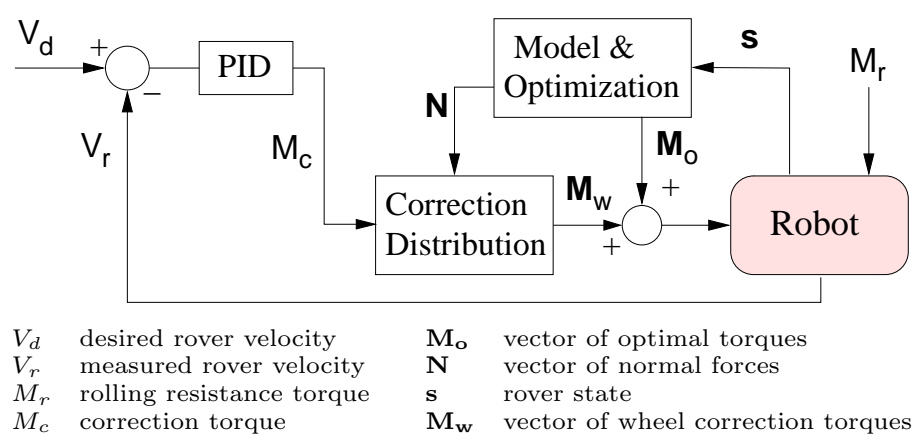

Fig. 5: Rover motion control loop.

The kernel of the control loop is a PID controller. It allows to estimate the additional torque to apply to each wheel in order to reach the desired rover's velocity $V_{d}$ and thus, minimizes the error $V_{d}-V_{r} . M_{c}$ is actually an estimate of the global rolling resistance torque $M_{r}$, which is considered as a perturbation by the PID controller. The rejection of the perturbation is guaranteed by the integral term $I$ of the PID. We assume that the rolling resistance is proportional to the normal force, thus the individual corrections for the wheels are calculated by

$$
M_{w_{i}}=\frac{N_{i}}{N_{m}} \cdot M_{c}
$$

where $N_{i}$ is the normal force on wheel $i$ and $N_{m}$ the average of all the normal forces. The derivative term $D$ of the PID allows to account for non modeled dynamic effects and helps to stabilize the system. The parameters estimation for the controller is not critical because we are more interested in minimizing slip than in reaching the desired velocity very precisely. For locomotion in rough terrain, a residual error on the velocity can be accepted as long as slip is minimized.

\section{Experimental results}

A simulation phase using Open Dynamics Engine ${ }^{2}$ has been initiated in order to test the approach and verify the theoretical concepts and assumptions. The

\footnotetext{
2 this library simulates rigid body dynamics in three dimensions, including advanced joint types and collision detection with friction.
} 
simulation parameters have been set as close as possible to the real operation conditions. However, the intent is not to get exact outputs but to compare different control strategies and detect/solve potential implementation problems. In the experiments, wheel slip has been taken as the main benchmark and the performance of our controller (predictive) has been compared to the controller presented in [6] (reactive). The reactive controller implements speed control (spd) for the wheels whereas torque control (trq) is used in our approach.

Three dimensional surfaces are used for the experiments (see Fig. 6). Because the trajectory of the rover depends on the control strategy, we consider an experiment to be valid if the distance between the final positions of both paths is smaller than $0.1 \mathrm{~m}$ (for a total distance of about $3.5 \mathrm{~m}$ ). This distance is small enough to allow performance comparison. For all the valid experiments, predictive control showed better performance than reactive control. In some cases the rover was even unable to climb some obstacles and to reach the final distance when driven using the reactive approach. It is interesting to note that the slip signal is scaled down for each wheel when using predictive control. Such behavior can be observed in Fig. 7: the peaks are generally at the same places for both controllers but the amplitude is much smaller for the reactive controller. Another interesting result is that the difference between the two methods increases when the friction coefficient gets lower. In other words, the advantage of using torque control becomes more and more interesting as the soil gets more slippery. Such a controller improves the climbing

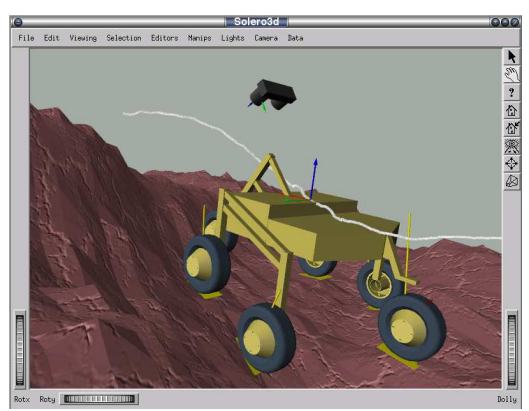

Fig. 6: Simulation environment

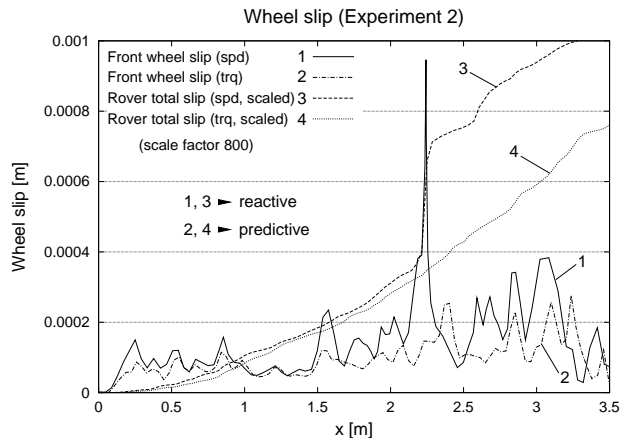

Fig. 7: Wheel slip

capabilities of the rover and limits wheel-slip, which in turn improves the accuracy of odometry. This way, it contributes to better position tracking in rough terrain. Furthermore, our approach can be adapted to any kind of wheeled rover and the needed processing power remains relatively low, which makes online computation feasible. Finally, the simulations show promising results and the system is mature enough to be implemented on SOLERO for real experiments. 


\section{Sensor fusion}

In our approach an Extended Information Filter (EIF) is used to combine the information acquired by the sensors. This formulation of the Kalman filter has interesting features: its mathematical expression is well suited to implement a distributed sensor fusion scheme and allows for easy extension of the system in order to accommodate any number of sensors, of any kind. Fig. 8 depicts the schematics of the sensor fusion process.

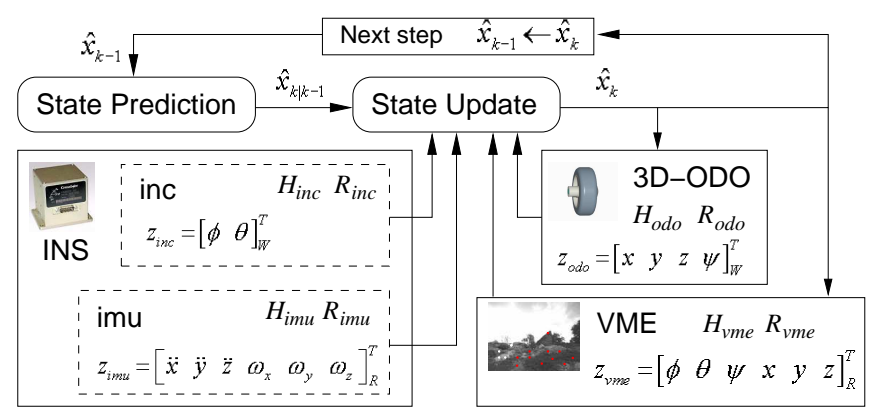

Fig. 8: Sensor fusion scheme

Sensor models: The position, velocity and attitude can be computed by integrating the measurement acquired by the IMU. However, the accelerometers and gyros are influenced by bias errors. In order to limit an unbounded growth of the error of integrated measurements, we have introduced biases in the model for the gyros $\left(b_{\omega x}, b_{\omega y}, b_{\omega z}\right)$ and the accelerometers $\left(b_{a x}, b_{a y}, b_{a z}\right)$. Unlike the roll and pitch angles, the rover's heading is not periodically updated by absolute data. Therefore, in order to limit the error growth, a special provision is included in the z-gyro model: a more accurate modeling, incorporating the scaling error $\Delta_{\omega z}$.

The robot used for this research is a partially skid-steered rover and the natural and controlled motion is mainly in the forward direction. Thus, the motion estimation errors due to wheel slip and wheel diameter variations have much more effect in the $\mathrm{x}-\mathrm{z}$ plane of the rover than along the transversal direction y. Therefore, scaling errors $\Delta_{o x}$ and $\Delta_{o z}$, modeling wheel slip and wheel diameter change, have been introduced only for the $\mathrm{x}$ and $\mathrm{z}$-axes. The error model for the odometry is tedious to develop because the robot is subject to drive across various types of terrains. In order to avoid terrain classification and complex wheel soil interaction modeling, we set the variance of the odometry as being proportional to the acceleration undergone by the rover. Indeed, slip mostly occurs in rough terrain, when negotiating an obstacle, while the robot is subject to accelerations. Similarly, the variance for the yaw angle has been set proportional to the angular rate. More details about the models of 
the IMU and 3D-Odometry can be found in [11] and reference [12] presents the error model associated to the estimations of VME.

State prediction model: The angular rates, biases, scaling errors and accelerations are random processes which are affected by the motion commands of the rover, time and other unmodeled parameters. However, they cannot be considered as pure white noise because they are highly time correlated. Instead, they are modelled as first order Gauss-Markov processes. Such modeling of the state transition allows to both consider the time correlation and to filter noise of the signals.

\section{Experimental results}

In order to better illustrate how each sensor contributes to the pose estimation and in which situation, the experiments have been divided into two parts. The first part describes the results of sensor fusion using inertial sensor and 3DOdometry only, whereas the second part involves all the three sensors i.e. 3D-Odometry, inertial sensor and VME.

Inertial and 3D-Odometry: The experimental results show that the inertial navigation system helps to correct odometric errors and significantly improves the pose estimate. The main contributions occur locally when the robot overcomes sharp-shaped obstacles (Fig. 9) and during asymmetric wheel slip. The improvement brought by the sensor fusion becomes more and more pronounced as the total path length increases. More results are presented in [11].
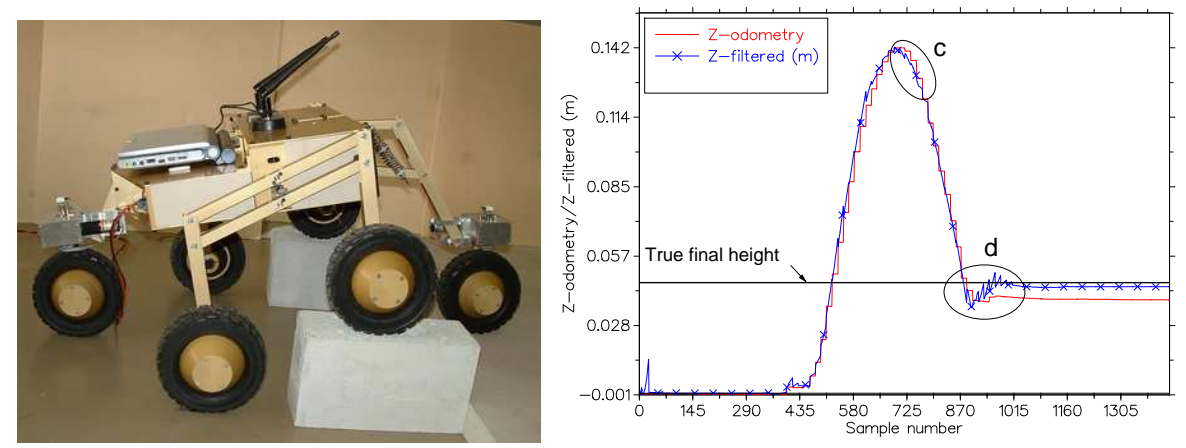

Fig. 9: Sensor fusion with 3D-Odometry and inertial sensors. The ellipses emphasis local corrections of the $\mathrm{z}$ coordinate.

Enhancement with VME: In the previous tests, only proprioceptive sensors have been integrated to estimate the robots position. Even if the inertial sensor helps to correct odometric error, there are situations where this combination of 
sensors does not provide enough information. For example, the situation where all the wheels are slipping is not detected by the system. In this case, only the odometric information is integrated, which produces erroneous position estimates. Thus, in order to increase the robustness of the localization and to limit the error growth, it is necessary to incorporate exteroceptive sensors. In this application, we use visual motion estimation based on stereovision[12].
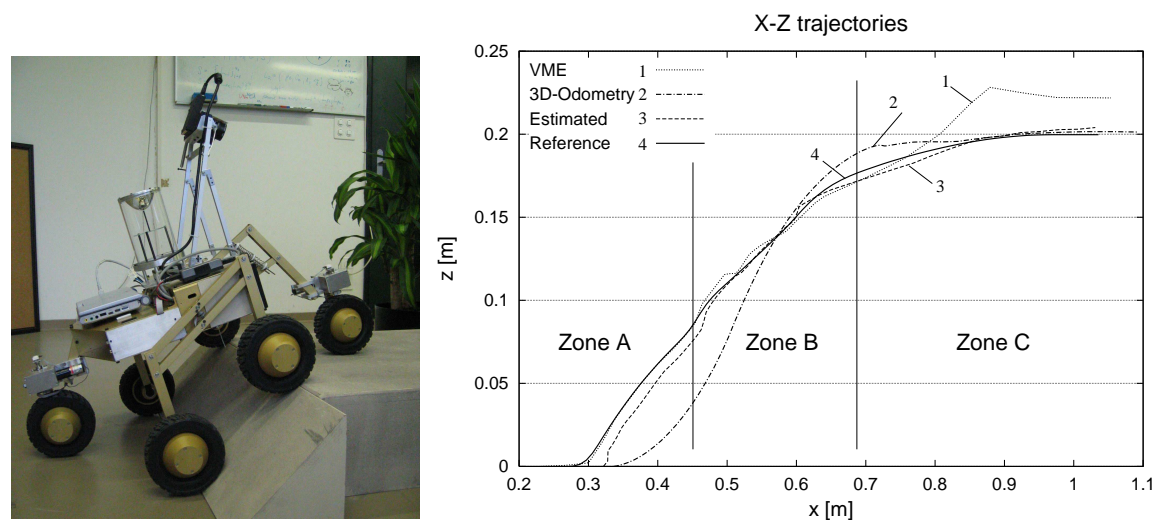

Fig. 10: Sensor fusion using 3D-Odometry, IMU and VME

In general, VME produces better estimates than the other sensors (but at a much slower rate). In particular, its estimates allow to correct the accumulated error due to wheel slip between two updates. However, in zone C (Fig. 10), less than thirty features have been matched between three subsequent images. The difficulty to find matches between these images is due to a high discrepancy between the views: when the rear wheel finally climbs the obstacle, it causes the rover to tilt forward rapidly. As a consequence, VME provided bad motion estimates with a high uncertainty. In this situation, less weight is given to VME and the sensor fusion could perfectly filter this bad information to produce a reasonably good estimate using 3D-Odometry and IMU instead. Finally, the estimated final position is very close to the measured final position. A final error of four millimeters for a trajectory longer than one meter $(0.4 \%)$ is very satisfactory, given the difficulty of the terrain.

\section{Conclusion}

This paper showed how 3D position tracking in rough terrain can be improved by considering the specificities of the vehicle used for locomotion. 3D-Odometry produces much better estimates than the standard approach because it takes the kinematics of the rover into account. Similarly, by considering a physical model of the chassis it is possible to minimize wheel-slip, 
which in turn contributes towards better localization. In rough terrain, the controller presented in Sect. 4 performs better than a controller based on a reactive approach. Finally, experimental results of sensor fusion involving 3DOdometry, inertial sensors and visual motion estimation have been presented. They prove that the use of complementary sensors improves the accuracy and the robustness of the motion estimation. In particular, the system was able to properly discard inaccurate visual motion information.

\section{References}

1. Strelow D, Singh S (2003) Online Motion Estimation from Image and Inertial Measurements, The 11th International Conference on Advanced Robotics, Portugal

2. Nebot E, Sukkarieh S, Durrant-Whyte H (1997) Inertial navigation aided with GPS information, In the proceedings of the Fourth Annual Conference of Mechatronics and Machine Vision in Practice

3. Siegwart R, Lamon P, Estier T, Lauria M, Piguet R (2000) Innovative design for wheeled locomotion in rough terrain, Journal of Robotics and Autonomous Systems, Elsevier, vol 40/2-3 p151-162

4. Lacroix S, Mallet A, Bonnafous D, Bauzil G, Fleury S, Herrb M, Chatila R (2002) Autonomous rover navigation on unknown terrains: functions and integration, International Journal of Robotics Research

5. Lamon P, Siegwart R (2003) 3D-Odometry for rough terrain - Towards real 3D navigation, IEEE International Conference on Robotics and Automation, Taipei, Taiwan

6. Baumgartner E.T, Aghazarian H, Trebi-Ollennu A, Huntsberger T.L, Garrett M.S (2000) State Estimation and Vehicle Localization for the FIDO Rover, Sensor Fusion and Decentralized Control in Autonomous Robotic Systems III, SPIE Proc. Vol. 4196, Boston, USA

7. Iagnemma K, Dubowsky S (2000) Mobile robot rough-terrain control (RTC) For planetary exploration, Proceedings ASME Design Engineering Technical Conferences, Baltimore, Maryland, USA

8. Yoshida K, Hamano H, Watanabe T (2002) Slip-Based Traction Control of a Planetary Rover, In the proceedings of the 8th International Symposium on Experimental Robotics, ISER, Italy

9. Iagnemma K, Shibley H, Dubowsky S (2002) On-Line Terrain Parameter Estimation for Planetary Rovers, IEEE International Conference on Robotics and Automation, Washington D.C, USA

10. Lamon P, Siegwart R (2005) Wheel torque control in rough terrain - modeling and simulation, IEEE International Conference on Robotics and Automation, Barcelona, Spain, in press

11. Lamon P, Siegwart R (2004) Inertial and 3D-odometry fusion in rough terrain Towards real 3D navigation, IEEE/RSJ International Conference on Intelligent Robots and Systems, Sendai, Japan

12. Jung I-K, Lacroix S (2003) Simultaneous Localization and Mapping with Stereovision, International Symposium on Robotics Research, Siena 